\title{
Source credibility and syllogistic reasoning
}

\author{
David E. Copeland • Kris Gunawan • \\ Nicole J. Bies-Hernandez
}

Published online: 6 November 2010

(C) Psychonomic Society, Inc. 2010

\begin{abstract}
Two experiments examined whether a source credibility effect would be observed for a syllogistic reasoning task. In the experiments, people were given two statements, presented as the results from a survey, followed by a conclusion that was supposedly made by one of two sources. In Experiment 1, one of the sources was described as honest and the other as dishonest, and in Experiment 2, one of the sources was described as an expert and the other as a non-expert. Because a pilot experiment showed that credibility can be overridden by people's experience with a source, all conclusions in Experiments 1 and 2 were ones that were likely to be accepted (i.e., necessary and possible strong conclusions). Both experiments showed a clear source credibility effect, particularly for the invalid conclusions. These results, along with the belief bias effect and previous research with conditional reasoning, suggest that people can be influenced by extraneous context, such as the honesty or expertise of a source, in a syllogistic reasoning task.
\end{abstract}

Keywords Source credibility Syllogistic reasoning .

Syllogism $\cdot$ Honesty $\cdot$ Expertise

\section{Introduction}

When people encounter information, they can evaluate the information based on its own merits or they can consider additional information, such as the source of that informa-

D. E. Copeland $(\bowtie) \cdot K$. Gunawan $\cdot$ N. J. Bies-Hernandez Department of Psychology, University of Nevada, Las Vegas, 4505 Maryland Pkwy Box 5030,

Las Vegas, NV 89154, USA

e-mail: david.copeland@unlv.edu tion. For example, people are more likely to treat information more favorably coming from a source that has been reliable over time than from a source that has been shown to be less than reliable (e.g., Gordon, Franklin, \& Beck, 2005). The idea of source credibility has been examined in numerous studies, primarily in studies examining persuasion (e.g., Petty \& Briñol, 2008; Petty \& Wegener, 1998). However, for some tasks, such as logical reasoning tasks, conclusions must be determined to be necessarily correct (i.e., logically valid) based solely on the logical argument. That is, people are supposed to evaluate information based on logic alone and should not rely on other factors such as source credibility. Research with conditional reasoning has shown evidence of this with expertise (Stevenson \& Over, 2001), and the goal of our study was to examine whether the credibility of a source, in regards to honesty or expertise, would influence people's performance on a categorical syllogism task.

A categorical syllogism is a deductive logic problem in which people are presented with two statements, or premises, which are assumed to be true. These statements relate three different terms or concepts categorically to one another, using the following quantifiers: all (A), none (E), some (I), and some...not $(\mathrm{O})$. In an abstract sense, the three terms can be generically referred to as $\mathrm{A}, \mathrm{B}$, and $\mathrm{C}$, or $\mathrm{X}, \mathrm{Y}$, and Z. Syllogisms can also use nonsense terms (e.g., zops, bips, and ruks) or everyday terms (e.g., artists, bakers, and cyclists). Regardless of the types of terms used in a syllogism, the first premise relates two of the terms ( $\mathrm{A}$ and $\mathrm{B}$ ) and the second premise relates one of the terms from the first premise (B) with a third term (C). For example, two premises might state the following:

Some of the artists are bakers

All of the bakers are cyclists 
The order in which the terms appear is referred to as the syllogistic figure, and there are four possible syllogistic figures: (1) AB-BC, (2) BA-CB, (3) AB-CB, and (4) BA$\mathrm{BC}$. It is common for syllogisms to be referred to using the quantifier abbreviations followed by the syllogistic figure. The example above would be abbreviated as IA1 because the first premise uses the quantifier some (I), the second premise uses all (A), and the terms are organized in the first syllogistic figure (i.e., AB-BC).

The actual task when presented with a syllogism is to either produce a conclusion (e.g., Johnson-Laird \& Bara, 1984), select from multiple choices (e.g., Copeland \& Radvansky, 2004), or to verify a conclusion that is presented (e.g., Evans, Handley, Harper, \& Johnson-Laird, 1999). Regardless of the format, people must determine that the conclusion is a necessary conclusion that relates the two terms that are only mentioned once in the premises (A and C). A necessary, or valid, conclusion is one that is true under all possible logical interpretations of the premises. For the earlier example, there are two acceptable conclusions, "Some of the artists are cyclists" or "Some of the cyclists are artists". All other conclusions for this syllogism would be considered invalid. For example, the conclusion "Some of the artists are not cyclists" is possible (i.e., one could represent a scenario where this is true), but does not hold true for every possible way to represent this scenario. The conclusion for a syllogism is abbreviated by signifying the quantifier with a capital letter and the order of the terms with lower case letters. In the earlier example, the two valid conclusions would be abbreviated as Eac and Eca. Finally, it should also be noted that some syllogisms have no valid conclusion. These syllogisms are typically abbreviated as NVC.

Over the years, numerous studies have shown both the positive and negative sides of humans' ability to reason logically (e.g., Johnson-Laird, 2006). On the one hand, studies have shown that people can reach accuracy levels near $90 \%$ on a syllogistic reasoning task (e.g., Copeland \& Radvansky, 2004). In terms of particular syllogisms, there are a set of syllogisms that are consistently easier to solve than others (typically referred to as one model syllogisms according to mental model theory; Johnson-Laird \& Bara, 1984), with performance typically exceeding $80 \%$ for these syllogisms. Also, people have been shown to successfully reason with unique quantifiers, such as "most", "few" (Chater \& Oaksford, 1999), and "only" (Johnson-Laird \& Byrne, 1989). On the other hand, studies have shown that people can be poor reasoners. For example, in most studies of syllogistic reasoning, people typically respond with (or select) the correct conclusion less than $50 \%$ of the time (e.g., Copeland \& Radvansky, 2004). One mistake that people make is that they tend to erroneously produce or accept conclusions to syllogisms that have no valid conclusion. Another mistake that people make is that they sometimes fail to see the correct way to interpret or represent logical quantifiers (e.g., Roberts, Newstead, \& Griggs, 2001). Also, people frequently do not consider testing whether possible or plausible conclusions hold up to scrutiny (Newstead, Handley, \& Buck, 1999).

One of the most well known ways in which people can be led astray in a logical reasoning task is the belief bias effect (Evans, Barston, \& Pollard, 1983). The belief bias effect is a pattern where people show a tendency to accept conclusions because they are believable, not because they are necessarily true. For example, a conclusion of "all polar bears have white fur" is more likely to be accepted than a conclusion of "all polar bears have black fur". The belief bias effect tends to occur more frequently for invalid than valid syllogisms (e.g., Ball, Phillips, Wade, \& Quayle, 2006). That is, while there tends to be a difference in the frequency of acceptance for believable and non-believable conclusions that are valid, people generally accept valid conclusions the majority of the time. However, for invalid conclusions, people rarely accept non-believable conclusions but regularly accept believable ones. Recent explanations state that the belief bias effect is a result of a dual-process framework: System 1 relies on a heuristic process and System 2 is a more analytic process (Evans, 2006; Evans \& CurtisHolmes, 2005; Stupple \& Ball, 2008). The belief bias effect is associated with the heuristic process, which is fast and implicit. In contrast, the analytic process, related to more deliberate reasoning, is slower, explicit, and subject to the constraints of working memory. This dual-process explanation has been supported by individual differences research showing that people higher in general intelligence are better able to resist the belief bias effect (Stanovich \& West, 1997).

A related, yet different, idea that has yet to be explored for syllogistic reasoning is the effect of source credibility (Chaiken \& Maheswaran, 1994; Petty \& Wegener, 1998; Pornpitakpan, 2004). Source credibility is based on the idea that people judge information not only on its own merits, but also by people's impressions of the individual who provides the information. Generally speaking, sources that are considered credible are more likely to persuade people than sources that are not credible (although, see Tormala, Briñol, \& Petty, 2006 for a reversal of this effect). This has been demonstrated for sources that are honest versus sources who are dishonest (Mills \& Jellison, 1967; Priester \& Petty, 1995), as well as sources who are experts versus sources who are not (DeBono \& Harnish, 1988; Rhine \& Severance, 1970). These studies have shown that people tend to be persuaded by sources that are more honest as well as sources that have more expertise. While the effects of source credibility have primarily been investigated in social psychology topics such as persuasion (Petty \& Briñol, 2008) and consumer choices (Goldsmith, Lafferty, 
\& Newell, 2000), recent cognitive research has shown effects of source credibility on source monitoring and memory (Gordon et al., 2005), as well as inference-making from text (Sparks \& Rapp, in press). If the inferencemaking process is similar for cognitive tasks such as text comprehension and reasoning (Radvansky \& Copeland, 2004), then it seems reasonable to expect that source credibility may affect reasoning performance.

Some studies have examined the effects of sources on areas of logical reasoning such as conditional reasoning (i.e., if $\mathrm{X} \rightarrow$ then Y). For example, Stevenson and Over (2001) showed that people were more likely to accept conclusions attributed to an expert than a novice. Other studies have shown that a source's personality can influence inference acceptance (Demeure, Bonnefon, \& Raufaste, 2009); also, a source's level of power (e.g., a statement from a child's father is perceived as a more powerful source than a statement from a child's brother) (Kilpatrick, Manktelow, \& Over, 2007) can influence the likelihood of accepting deontic conclusions. Along this line, work by Hilton, Kemmelmeier, and Bonnefon (2005) has demonstrated that the goal of the information conveyed in conditional premises, such as whether it is meant as an instruction or a hypothetical, can influence acceptance. It has even been shown that people are more likely to believe specific aspects of a conditional statement than others (e.g., believing the antecedent more than the consequent) (Hadjichristidis, Handley, Sloman, Evans, Over, \& Stevenson, 2007). These studies clearly demonstrate that the conditional reasoning process can be affected by context (Evans, 2002).

At this point, however, there has been only one study that has examined the effect of source credibility on categorical (i.e., syllogistic) reasoning. Bettinghaus, Miller, and Steinfatt (1970), in an examination of the effects of dogmatism on reasoning (i.e., whether people scoring high or low on a measure of dogmatism performed different on a reasoning task), considered how a number of factors affect reasoning performance. While the primary interest was in the effects of dogmatism, the study also considered the source of information as well as a manipulation of the content (i.e., belief bias effects). This study showed no clear effects of source credibility on reasoning performance; the only effect of source that was observed was as part of an interaction with the other factors. There are a number of reasons why no effect was observed. First, very few syllogisms were used in this experiment; only four were presented in the experiment. This can be problematic because there is a large amount of variability in the difficulty of syllogisms. Second, the presented syllogisms were very wordy (one premise was over 30 words in length) and, thus, the structure of the syllogism may not have been clear. For comparison, in most studies of syllogistic reasoning, including our study, premises typically contain four to nine words.
Third, real people were used as sources, such as Billy Graham and former President Dwight Eisenhower. The fact that people had prior knowledge about the sources could influence their evaluation of whether the source was credible or not. Similarly, the syllogisms were presented with political content (e.g., support for the Vietnam War); as mentioned earlier, prior beliefs about content have clearly been shown to influence reasoning (Evans et al., 1983).

In our study, the effect of source credibility was examined across two experiments. Previous studies in social psychological research that have examined source credibility have typically presented sources that varied either in their trustworthiness (e.g., Priester \& Petty, 1995) or in their level of expertise (e.g., DeBono \& Harnish, 1988). While previous studies in social research have shown that people are more accepting of information that comes from an honest source than from a dishonest source, it is unclear whether honesty will have an effect on categorical reasoning. Because drawing conclusions to categorical syllogisms can be difficult, participants may not think that honesty is a factor that would affect accuracy. That is, even if a source is thought to be honest, that source still may not be able to accurately make the correct inference. Thus, it is possible that honesty may not have an effect on people's acceptance of conclusions. Instead, participants may be more likely to accept a conclusion made by someone who is an expert at drawing conclusions than someone who is not. In our study, Experiment 1 examined the source credibility effect based on the honesty of sources and Experiment 2 examined the source credibility effect based on the expertise of sources.

\section{Experiment 1}

In Experiment 1, participants were presented with categorical syllogisms using a verification task. The syllogism task was presented under the guise that the premises were facts based on a survey conducted in a small town, and the conclusions for each syllogism were inferences made by one of two people who worked in the town, an honest or dishonest individual. Participants were asked to verify whether each inference was definitely true, assuming the facts from the survey were true. Experiment 1 used valid inferences for half the trials and invalid inferences for the remaining trials. The valid and invalid syllogisms were based on syllogisms classified as necessary and possible, respectively, by Evans et al. (1999). Necessary refers to the fact that no matter what representation one creates for that syllogism, that particular conclusion is true. Possible refers to the idea that it is possible to create a representation where that conclusion seems true; however, the conclusion does not hold true if alternative representations are created. Thus, for half of the trials the correct response was to accept the inference, and 
for the other half the correct response was to not accept the inference. The reason that possible syllogisms were used for the invalid trials was because a pilot study revealed that there was no source credibility effect when impossible conclusions (i.e., conclusions that were not true for any representation) were used $^{1}$. This is most likely because, even though people are provided with positive information about one of the sources (i.e., the honest one), people's impressions of the sources may be overridden by their experience (Gordon \& Bryant, 2010). That is, when for half of the trials both sources seem to be clearly wrong (i.e., the impossible conclusions), then this may reduce both sources' credibility. To ensure that these possible conclusions seemed reasonable, we selected possible strong conclusions as labeled by Evans et al. (1999). "Strong" refers to the fact that many people are likely to mistakenly accept these conclusions as valid. Thus, all conclusions should at least seem reasonable to accept for the participants. This should increase the likelihood that participants maintain their belief that one source is more credible than the other.

Based on previous research that has examined the effects of source credibility (e.g., Priester \& Petty, 1995), it was possible that people would be more likely to accept inferences from the honest source, regardless of whether the inference was valid. Research with conditional reasoning has shown that people can be influenced by context (Evans, 2002). More importantly, there have been specific studies that have shown effects based on the source of information (e.g., Stevenson \& Over, 2001). Together, these studies and research from social psychology suggest that a source credibility effect would be observed in Experiment 1. A source credibility effect would also be consistent with a number of different theories that are based on a dualprocess framework (Evans, 2007). For example, theories based on pre-emptive conflict resolution (e.g., Evans et al., 1983) and default-interventionist models (e.g., Evans, 2006), sometimes referred to as belief-first models (e.g., Stupple \& Ball, 2008), would predict that conclusions attributed to an honest source, which would be more believable, would be based on System 1 (heuristic) processing (i.e., conclusions from the honest source are likely to be accepted because those conclusions are believed to be more truthful). In contrast, conclusions from the dishonest source would be more likely to use System 2

\footnotetext{
${ }^{1}$ In the pilot experiment, a 2 (necessary vs. impossible) $\times 2$ (honest vs. dishonest) repeated measures analysis of variance (ANOVA) was conducted with accuracy as the dependent variable. There was a main effect of syllogism, $F(1,59)=34.06, M S E=0.02, p<0.001, \eta_{p}{ }^{2}=0.37$, with participants being less accurate for the necessary $(M=0.82, S E=$ $0.02)$ than the impossible syllogisms $(M=0.92, S E=0.01)$. However, there was no significant difference in accuracy between the honest $(M=$ $0.88, S E=0.02)$ and dishonest $(M=0.87, S E=0.02)$ sources, nor was there a significant interaction, both $F S<1$.
}

(analytical) processing. The latter idea suggests that people should be likely to accept necessary conclusions from dishonest sources, but should be more likely to reject invalid (in this case, possible strong) conclusions from dishonest sources than invalid conclusions from honest sources. In other words, people are more likely to use a falsifying approach for the dishonest source (Klauer, Musch, \& Naumer, 2000). Other dual-process accounts, such as computational escape hatch models (sometimes referred to as reasoning-first models; Stupple \& Ball, 2008), would predict that people would use System 1 "when a conclusion is possible but not necessitated by the premises" (Stupple \& Ball, 2008, p. 171). In other words, for the necessary conclusions, people should rely on System 2, and thus, be likely to accept most conclusions regardless of source. However, for possible strong conclusions, people relying more on System 1 should be more likely to accept conclusions from the honest source and reject conclusions from the dishonest source. Thus, regardless of the dualprocess account, accuracy should be high for necessary conclusions. For possible strong conclusions, though, accuracy should be better for the dishonest source because people are more likely to reject those conclusions. The pre-emptive conflict resolution and default-interventionist explanations predict it to be a result of analytical processing, while the computational escape hatch explanation predicts that it would be a result of heuristic-based processing.

It should also be considered that, similar to the pilot experiment, there could be no effect of source credibility in Experiment 1. There are two reasons why this pattern might be observed. First, because syllogisms are based on logical deduction, people may disregard all information about the source and instead simply evaluate the inferences using a reasoning process such as mental models (e.g., JohnsonLaird \& Bara, 1984). That is, people may recognize these categorical syllogisms as requiring the use of deductive logic, and may employ the more analytical part of the dualprocess framework (i.e., System 2) regardless of the source. This is a possibility because participants may believe that good intentions may not be enough to draw an inference that is correct. Thus, just because an individual is honest may not be enough to cause participants to trust the inferences made by that person. The second reason is based on the idea that if people primarily rely on analytical reasoning processes (i.e., System 2) and they are successful at recognizing that for half of the trials both sources seem to be wrong (as in the pilot experiment), then this may reduce the honest source's credibility. In other words, their impressions of the sources may be overridden by their experience (Gordon \& Bryant, 2010).

In addition to participants' accuracy on the syllogism task, Experiment 1 also included a second dependent measure. At the end of the syllogistic reasoning task, 
participants were asked to rate which source they thought was correct more often. The reason for including this question is that it was possible that there might not be a clear change in performance (i.e., no effect of source credibility on acceptance of conclusions). Even if the participants' behavior is not changed, it is possible that their memory of who was more accurate may be influenced by the source descriptions (Gordon et al., 2005).

\section{Method}

\section{Participants}

Fifty-two undergraduates (27 males and 25 females, mean age 19.87 years) from the University of Nevada, Las Vegas participated in exchange for partial course credit. The data from three additional participants was not included in the analyses (see explanation below).

\section{Materials and procedure}

At the beginning of the experiment, participants were seated at a computer and were presented with the following instructions:

You will be presented with reports about groups of people from the small town of River Village. Recently they conducted a survey of hobbies and attitudes (e.g., Do you play chess? What kind of computer do you use?) and all 200 people in the town responded. The first 2 statements in the report are facts that are based on results of the survey. At the end of each report there is a conclusion made by one of two people, Quentin or Zane, that is an interpretation of the facts in the report. Your job is to indicate whether the conclusion made by Quentin or Zane is correct. Before you begin, you will be given descriptions about Quentin and Zane. Please read this information carefully as you will be tested on it right after you read the description.

Following those instructions, people were presented with a biographical sketch of one person along with a threequestion quiz on the biographical information. This was followed by the biographical sketch and quiz for the other person. The biographical sketches were adapted from those used by Sparks and Rapp (in press). The order that the Quentin and Zane biographical sketches were presented was counterbalanced across participants. The biographical sketches of Quentin and Zane are listed below:

\section{QUENTIN CARTER}

Quentin Carter has been the River Village fire chief for 25 years. Whenever someone's home has been damaged by a severe storm, Quentin helps clean up the debris and pays for a portion of the repairs. Quentin is hardworking and willing to help those in need. Residents know that Quentin is honest and trustworthy.

\section{ZANE ANDERSON}

Zane Anderson has served as treasurer of River Village for 15 years. In the last election cycle, Zane convinced some of his campaign workers to solicit elderly voters for large donations. Zane then used the donations to buy himself a new sports car. Residents know that Zane is dishonest and untrustworthy.

The quiz questions were yes/no questions that asked about basic information, such as "Was Quentin known to help those in need?" or "Do the residents consider Zane to be untrustworthy?" People responded by pressing the "Y" or "N" key on the keyboard to answer "yes" or "no", respectively. The purpose of the quizzes was to ensure that people would better remember the characteristics of Quentin and Zane; namely, that Quentin was an honest person and Zane was a dishonest person. Accuracy scores were high and participants did not differ on their accuracy for the biographical sketches of Quentin $(M=0.97, S E=0.01)$ and Zane $(M=0.95$, $S E=0.02), F<1$.

Following the biographical sketches and quizzes, participants completed the main task. Here, participants were presented a total of 32 categorical syllogisms as a verification task. As a verification task, premises were presented along with a conclusion and participants had to determine if the presented conclusion was correct. Based on the classification of Evans et al. (1999), 16 of the syllogisms were rated as necessary and 16 were rated as possible strong. Eight of each type of syllogism had a conclusion with the terms in an a-c order ( 2 each from the 4 syllogistic figures) and the remaining eight had a conclusion with the terms in a c-a order (2 each from the 4 syllogistic figures). Also, for both the necessary and possible strong syllogisms, one quarter of the syllogisms were drawn from each syllogistic figure. A complete list of the 32 syllogisms that were used is in the Appendix. To make the syllogisms appear as if they were based on information from the town survey, all of the syllogisms were randomly assigned three hobby/status terms. The hobby/status terms were selected so that they did not combine to make statements that were necessarily true or false based on beliefs or popular culture. For example, because a combination such as "All cigarette smokers are joggers" is likely to be unbelievable to most people (i.e., cigarette smoking is very unhealthy while jogging is considered very healthy), combinations like this were removed and replaced by new hobby/status terms. An 
example of a necessary syllogism (with its corresponding conclusion) is listed below:

(IA1 - Ica)

Some married people are skiers

All skiers are tennis players

Some tennis players are married people

This is a necessary conclusion because this conclusion must follow, assuming the premises are true. The conclusion is classified as c-a because the final (non-linking) term from the premises is listed first in the conclusion. Below is an example of possible strong syllogism (with its corresponding conclusion):

$(\mathrm{AO} 1-\mathrm{Oac})$

All racquetball players are recyclers

Some recyclers are not cyclists

Some racquetball players are not cyclists

While this conclusion is possible, it is not logically necessary (i.e., there is no valid conclusion that can be drawn for this specific syllogism). For example, it is possible that the recyclers who are not cyclists are also not racquetball players; thus, there is no connection one can make between racquetball players and cyclists. This conclusion is classified as a-c because the initial (non-linking) term from the premises is listed first in the conclusion.

For each participant, half of the necessary and half of the possible strong syllogisms were associated with Quentin and the others were associated with Zane. This split into two sets maintained equal numbers of ac and ca conclusions, and equal numbers from each syllogistic figure. Using Johnson-Laird and Bara's (1984) classification of number of models as a rough measure of syllogism difficulty, the two sets of syllogisms (Set 1: $M=1.81$, $S E=0.19$; Set 2: $M=1.88, S E=0.18$ ) did not differ in terms of number of models, $F<1$. This matching process was counterbalanced across participants so that each syllogism was associated with Quentin and Zane an equal number of times. The conclusions were presented by preceding the actual conclusion with either, "(The fire chief) Quentin's conclusion:" or "(The treasurer) Zane's conclusion:". Participants were instructed to press the "Y" button on the keyboard if they thought the conclusion was correct or the "N" button on the keyboard if they thought the conclusion was incorrect. The syllogisms were presented in a different random order to each participant.

Finally, after completing the syllogism task, participants were presented with two brief questions. First, participants were asked to give their impression as to who was more accurate, Quentin or Zane. Participants made their response by using a scale from 1 to 7 . A rating of 1 indicated that Zane was clearly more accurate, 4 indicated that they were equally accurate, and 7 indicated that Quentin was clearly more accurate. The second question asked participants to identify which person was the honest one. This was done as a manipulation check to ensure that throughout the task that participants remembered which source was honest and which was not. The data from three participants who incorrectly responded with "Zane" as the honest one were not included in the analyses.

\section{Design}

For the syllogism task, a 2 (necessary vs. possible strong syllogism) × 2 (honest vs. dishonest source) repeated measures design was used with accuracy (i.e., \% correct) as the dependent variable. An effect of source credibility would be observed by one or both of the following: (1) for the necessary syllogisms: higher accuracy percentage for the honest source, (2) for the possible strong syllogisms: lower accuracy for the honest source (i.e., trusting the honest source for these invalid syllogisms would lead to worse performance). For the impression question, a score significantly greater than 4 (sources were equally accurate) would indicate that people thought the honest source was more accurate.

\section{Results and discussion}

\section{Accuracy}

A 2 (necessary vs. possible strong) $\times 2$ (honest vs. dishonest) repeated measures analysis of variance (ANOVA) was conducted with accuracy as the dependent variable (see Fig. 1). There was a main effect for the type of syllogism,

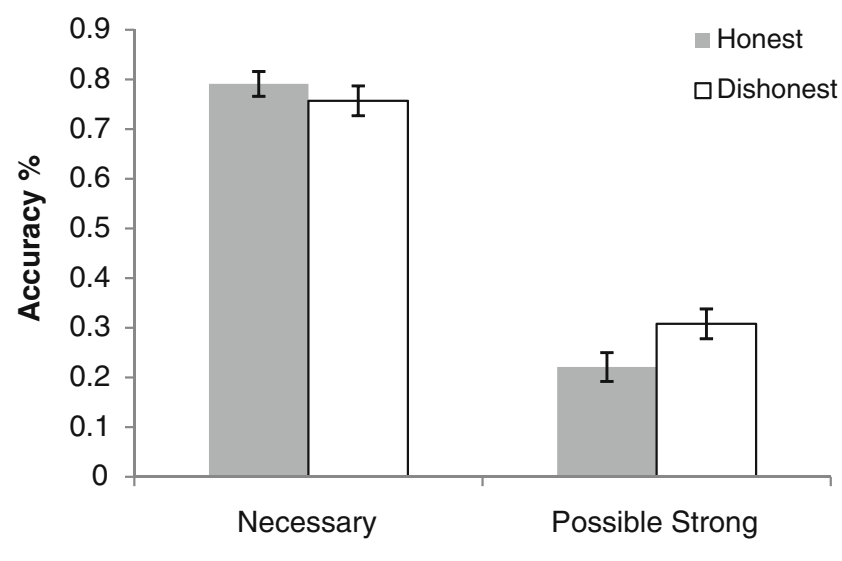

Type of Syllogism

Fig. 1 Mean accuracy performance in Experiment 1 for necessary and possible strong syllogisms based on whether the source was presented as honest or dishonest 
with higher accuracy for necessary $(M=0.77, S E=0.02)$ than possible strong $(M=0.26, S E=0.02)$ syllogisms, $F(1$, $51)=212.75, M S E=0.06, p<0.001, \eta_{p}{ }^{2}=0.81$. This was not surprising because, based on past studies (e.g., Evans et al., 1999), people should correctly accept necessary conclusions and should incorrectly accept possible strong conclusions. Although there was no significant difference for the honest $(M=0.51, S E=0.02)$ and dishonest source $(M=0.53, S E=0.02)$ in accuracy, $F(1,51)=1.62, M S E=$ $0.02, p=0.21, \eta_{p}{ }^{2}=0.03$, the interaction between syllogism and source was significant, $F(1,51)=4.43, M S E=0.04, p<$ $0.05, \eta_{p}{ }^{2}=0.08$. Follow-up tests showed that accuracy was better for the honest $(M=0.79, S E=0.03)$ than the dishonest $(M=0.76, S E=0.03)$ source for the necessary syllogisms, but this difference was not significant, $F<1$. For the possible strong syllogisms, accuracy was worse for the honest $(M=$ $0.22, S E=0.03)$ than the dishonest $(M=0.31, S E=0.03)$ source, $F(1,51)=6.26, M S E=0.03, p<0.05, \eta_{p}{ }^{2}=0.11$. Thus, while source credibility did not have a significant benefit on performance for the necessary syllogisms, it led to a decrement in performance for the possible strong syllogisms.

These results show that participants were more likely to accept conclusions from an honest source. In particular, follow-up tests showed that there was an interesting pattern in that there was a significant difference in conclusion acceptance for the possible strong syllogisms, but not for the necessary syllogisms. This is consistent with previous research with the belief bias effect that showed larger effects for invalid than valid syllogisms (Evans et al, 1983). Also, this pattern was consistent with predictions made by various theories based on a dual-process framework (Stupple \& Ball, 2008).

\section{Impression of credibility/accuracy}

As a reminder, for the impression of credibility/accuracy, participants used a scale from 1 to 7 to indicate which source they thought was more accurate. A rating of 1 indicated that Zane was clearly more accurate, a rating of 4 indicated that the two sources were equally accurate, and a rating of 7 indicated that Quentin was clearly more accurate. A one sample t-test showed that the rating $(M=$ 4.35, $S E=0.16)$ was significantly greater than $4, t(51)=$ 2.13, $p<0.05$. In other words, participants believed that Quentin (i.e., the honest one) was more accurate than Zane (i.e., the dishonest one). This is consistent with the pattern of responses that showed that people were more likely to accept Quentin's conclusions.

Together, both dependent measures used in Experiment 1 were consistent with a source credibility effect on reasoning performance. Conclusions made by the honest source were more likely to be accepted than conclusions made by the dishonest source. Furthermore, this effect was clearer for the invalid syllogisms, which is consistent with other effects such as belief bias (Ball et al., 2006). Finally, not only did source credibility influence people's responses, but source credibility also influenced people's memories, or impressions, of the sources. Even though the two sources were equally correct, people remembered that the honest source was more accurate than the dishonest source. This is consistent with previous memory research with source credibility (Gordon et al., 2005).

\section{Experiment 2}

There were two goals for Experiment 2. The first goal was to try to replicate the source credibility effect observed in Experiment 1. The second goal was to determine whether the source credibility effect would be present for a characteristic besides honesty (i.e., expertise). In Experiment 2, two sources (Quentin and Zane) were described as either (a) having experience and expertise conducting and interpreting surveys about human lifestyles or (b) working in some other unrelated profession (i.e., little to no experience drawing conclusions from survey data). Based on the results of Experiment 1, as well as previous studies examining source expertise and conditional reasoning (Stevenson \& Over, 2001), it was expected that participants would be more likely to accept conclusions from the expert.

\section{Method}

\section{Participants}

Sixty undergraduates (26 males and 34 females, mean age 21.02 years) from the University of Nevada, Las Vegas participated in exchange for partial course credit. None of these individuals participated in Experiment 1. One additional participant was not included in the analysis (see explanation below).

\section{Materials and procedure}

The materials and procedure for Experiment 2 were nearly identical to Experiment 1. The same initial instructions and syllogisms were used. The major difference for Experiment 2 was that the biographical sketches of Quentin and Zane were altered so that Quentin was described as an expert working with surveys and drawing conclusions whereas Zane was not an expert. Below are the biographical sketches used in Experiment 2:

\section{QUENTIN CARTER}

Quentin Carter has been a professor at the local university for 25 years. He has a degree in psychol- 
ogy from Harvard. Quentin conducts survey research on people's attitudes and lifestyles. He has analyzed and published over one hundred scientific papers and has also won numerous research awards. Quentin is well respected by his peers at the university.

\section{ZANE ANDERSON}

Zane Anderson has worked as a mechanic in town for 35 years. He played football in high school, but Zane did not have good enough grades to go to college. After graduation his father helped him get a job with a local garage. Zane is really good working with car engines and auto body work. Zane is thought of as a friendly guy around town.

As with Experiment 1, when tested on these biographical sketches, accuracy scores were high and there was no significant difference for their responses for Quentin $(M=$ $0.93, S E=0.02)$ and Zane $(M=0.96, S E=0.02), F(1,59)=$ $1.48, M S E=0.02, p=0.23, \eta_{p}{ }^{2}=0.03$.

The only other change to Experiment 2 was with the memory question asked at the end of the task. Instead of asking which person was more honest (as was done in Experiment 1), in Experiment 2, people were asked, "Which of the people from this study was considered to be an expert with analyzing surveys, Quentin (the professor) or Zane (the mechanic)?" One participant who incorrectly responded with "Zane" as the expert was not included in the data analyses.

\section{Design}

Similar to Experiment 1, a 2 (necessary vs. possible strong syllogism) x 2 (expert vs. non-expert source) repeated measures design was used with accuracy (i.e., $\%$ correct) as the dependent variable. A source credibility effect would be based on one or both of the following: (1) necessary syllogisms: higher accuracy percentage for the expert source, (2) possible strong syllogisms: lower accuracy for the expert source. For the impression question, a rating score significantly greater than 4 indicates that people thought the expert source was more accurate.

\section{Results and discussion}

Accuracy

A 2 (necessary vs. possible strong) x 2 (expert vs. nonexpert) repeated measures ANOVA was conducted, using accuracy as the dependent variable (see Fig. 2). As in

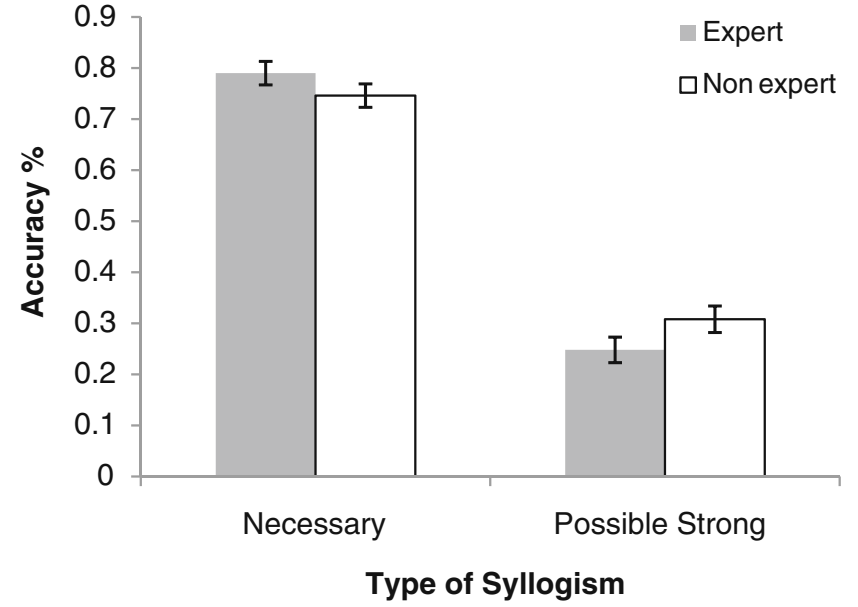

Fig. 2 Mean accuracy performance in Experiment 2 for necessary and possible strong syllogisms based on whether the source was presented as an expert or a non-expert

Experiment 1, there was a main effect for syllogism, with higher accuracy for necessary $(M=0.77, S E=0.02)$ than possible strong $(M=0.28, S E=0.02)$ syllogisms, $F$ $(1,59)=296.61, M S E=0.05, p<0.001, \eta_{p}{ }^{2}=0.83$. There was no difference for the expert $(M=0.52, S E=0.02)$ and non-expert $(M=0.53, S E=0.02)$ source in terms of accuracy, $F<1$. Importantly, the interaction between syllogism and source was significant, $F(1,59)=6.82$, $M S E=0.02, p<0.05, \eta_{p}{ }^{2}=0.10$. Consistent with Experiment 1, follow-up tests showed that for the necessary syllogisms accuracy was not significantly better for the expert $(M=0.79, S E=0.02)$ than the non-expert $(M=0.75, S E=0.02), F(1,59)=1.97, M S E=0.03, p=$ $0.17, \eta_{p}{ }^{2}=0.03$. For the possible strong syllogisms, accuracy was significantly worse for the expert $(M=0.25$, $S E=0.03)$ than the non-expert $(M=0.31, S E=0.03), F(1$, $59)=6.04, M S E=0.02, p<0.05, \eta_{p}{ }^{2}=0.09$. This is a similar pattern to the one that was observed in Experiment 1 and it supports the findings from Stevenson and Over's (2001) study of expertise and conditional reasoning.

Impression of credibility/accuracy

As a reminder, participants used a scale from 1 to 7 to indicate who they thought was more accurate $(1=$ Zane was clearly more accurate, $4=$ they were equally accurate, and $7=$ Quentin was clearly more accurate). A one sample $t$-test showed that the rating $(M=4.37, S E=0.13)$ was significantly greater than $4, t(59)=2.73, p<0.01$. Thus, participants believed that Quentin (i.e., the expert) was more accurate than Zane (i.e., the non-expert), which is consistent with their response patterns to the syllogisms. As with the accuracy results, this pattern replicates the one that was observed in Experiment 1. 


\section{General discussion}

Two experiments examined whether the credibility of a source would influence people's accuracy when verifying a conclusion for a categorical syllogism. In Experiment 1, two sources differed in terms of honesty (i.e., one source was described as honest and the other as dishonest). In Experiment 2, the two sources differed in terms of their expertise in working with survey data (i.e., one source was an expert with conducting and interpreting surveys and the other did not have any expertise). Both Experiments 1 and 2 revealed clear source credibility effects. These effects showed that people were more likely to accept conclusions that were made by a source who was either honest or an expert. This study adds to the existing social psychology literature on effects of source credibility (Petty \& Wegener, 1998; Pornpitakpan, 2004). In particular, the results of our study are consistent with studies showing source credibility effects based on characteristics such as honesty (Mills \& Jellison, 1967; Priester \& Petty, 1995) and expertise (DeBono \& Harnish, 1988; Rhine \& Severance, 1970).

Our study improved on an earlier study by Bettinghaus et al. (1970) that considered the idea of source credibility effects on syllogistic reasoning. While their study did not show any direct effects of source credibility, there were some flaws in the design that were addressed in our study. First, our study used a larger number of syllogisms; sixteen syllogisms were used for each condition in both experiments. Second, our study was careful to avoid presenting the syllogisms with potentially biasing information related to prior knowledge. Whereas the Bettinghaus et al. (1970) study used syllogisms with political information that could have been influenced by already held political beliefs or ideas, our study used general hobbies or interests that were not necessarily true or false based on beliefs or popular culture. Finally, our study presented the syllogisms in a straightforward manner without unnecessary text to ensure that people could easily identify the syllogistic premises.

The source credibility effect observed in our study adds to the existing findings that people are not completely logical (e.g., Evans, 2002). If people were basing their responses only on logical reasoning, then it would not matter which source made each conclusion. Instead, similar to what has been observed in conditional reasoning (e.g., Stevenson \& Over, 2001), our study showed that people were more likely to accept conclusions of categorical syllogisms from honest or expert sources. However, it is important to note that differences between a pilot experiment and Experiment 1 showed that people do not blindly use information that is provided to them and let it affect their performance on a reasoning task. The pilot experiment showed that what people experience for themselves can override initial impressions of source credibility. The fact that people can develop impressions of source credibility based on experience is consistent with recent cognitive research (e.g., Gordon \& Bryant, 2010). Our study showed that presenting impossible conclusions is enough to override the information people were told about the sources, but presenting possible strong conclusions that were invalid did not eliminate the source credibility effect. However, at this point it is unclear what amount of clearly inconsistent information is enough to diminish or eliminate the effect. Future studies could further address this point by manipulating the proportion of valid and invalid conclusions, as well as the types of invalid conclusions (i.e., possible strong, possible weak, and impossible).

The patterns observed here are similar to those that have been observed for the belief bias effect (Evans et al., 1983). Analogous to the belief bias effect, which shows that people are more likely to accept believable conclusions, the source credibility effect showed that people were more likely to accept conclusions from a trustworthy, or believable, source. The source credibility effect is similar to the belief bias effect in other ways too. Importantly, both seem to occur more frequently for invalid than valid syllogisms. For valid syllogisms, people are likely to accept the conclusion, regardless of the believability (e.g., Ball et al., 2006) or the source. However, for invalid syllogisms, conclusions that might not normally be accepted are more likely to be accepted if they are believable (e.g., Ball et al., 2006) or come from a credible source. The results of our study are consistent with the selective processing model explanation of the belief bias effect (Klauer et al., 2000; see also Evans, Handley, \& Harper, 2001) in that additional information or beliefs can influence the search for a mental model representation of a syllogism ${ }^{2}$. Specifically, context effects (e.g., differences between credible and non-credible sources) are small for valid syllogisms because any model that can be constructed will support the conclusion. However, for possible strong conclusions, while a credible source will lead people to construct a model that supports the conclusion, a non-credible source will lead people to search for a model that is not consistent with the conclusion which then leads to an increased likelihood of rejecting the conclusion. In addition, this explanation may also account for the lack of effect for clearly invalid (i.e., impossible) syllogisms that were used in the pilot experiment. Here, regardless of whether people are trying to construct a model that supports the presented conclusion (i.e., credible source) or construct a model that does not support it (i.e., noncredible source), no models will be found that can support the conclusion; thus, no credibility effect is observed.

\footnotetext{
${ }^{2}$ We would like to thank the anonymous reviewers for their suggestions concerning this explanation as well as others related to the dual process accounts of reasoning.
} 
The findings from our study are also consistent with a dual-process framework (Chaiken \& Maheswaran, 1994; Evans, 2006; Evans \& Curtis-Holmes, 2005; Stupple \& Ball, 2008). Different dual-process approaches, such as the pre-emptive conflict resolution (Evans et al., 1983), defaultinterventionist (Evans, 2006), and computational escape hatch models (e.g., see Stupple \& Ball, 2008), predicted a pattern of results where accuracy performance for the necessary conclusions would be high for both sources, but performance would differ for the possible strong conclusions depending on the source, namely, that accuracy would be better for the dishonest source. While they make the same predictions, these different explanations of the dualprocess framework describe different paths to these outcomes. The pre-emptive conflict resolution and defaultinterventionist models explain the higher likelihood of rejecting possible strong conclusions attributed to the dishonest source as a result of analytical (i.e., System 2) processing, while the computational escape hatch models attribute it to heuristic-based processing (i.e., System 1). However, the accuracy data from our study is limited in that it does not allow us to distinguish between these approaches. Future studies with categorical syllogisms could further explore the source credibility effect and dual-process accounts by either examining other types of dependent measures (Evans, 2007), such as response times (e.g., Stupple \& Ball, 2008), or requiring rapid responses (e.g., Evans \& Curtis-Holmes, 2005).

\section{Conclusion}

In summary, this study demonstrated that source credibility can affect reasoning performance for a categorical syllogism task. Experiments 1 and 2 showed that these effects can be observed for sources differing in honesty or expertise, respectively. While these effects were smaller than effects observed for the belief bias effect, the patterns were consistent with the belief bias effect. In particular, there was a larger effect for invalid than valid syllogisms (e.g., Ball et al., 2006). In addition, secondary dependent measures were consistent with an earlier study (Gordon et al., 2005) that showed that memory of a source's accuracy can be influenced by credibility.

Acknowledgments We would like to thank Hilary Billings, Jenny Bivens, Hailey Connor, Jason Kelsey, Clinton Kyle, Joshua Molina, Sam Montano, Ashley Moreno, Nevenka Olivari and Abby Rotstein for their assistance in collecting the data. We would also like to thank three anonymous reviewers for their very helpful comments and suggestions on earlier versions of this manuscript. This project was not supported by any external funding sources.

\section{Appendix}

Table 1 Syllogisms with their corresponding conclusions used in Experiments 1 and 2

\begin{tabular}{llll}
\hline Necessary syllogisms & \multicolumn{2}{l}{ Possible strong syllogisms } \\
\hline AA1 & Aac & AO1 & Oac \\
AE1 & Eac & II1 & Iac \\
AI2 & Iac & AO2 & Oac \\
IE2 & Oac & IA2 & Iac \\
AE3 & Eac & IA3 & Iac \\
EA3 & Eac & IO3 & Oac \\
AI4 & Iac & AA4 & Aac \\
AE4 & Oac & OA4 & Oac \\
IA1 & Ica & AI1 & Ica \\
EA1 & Oca & IO1 & Oca \\
AA2 & Aca & II2 & Ica \\
AI2 & Ica & IO2 & Oca \\
EA3 & Eca & AI3 & Ica \\
EI3 & Oca & IA3 & Ica \\
AI4 & Ica & IO4 & Oca \\
EI4 & Oca & OI4 & Oca \\
\hline
\end{tabular}

\section{References}

Ball, L. J., Phillips, P., Wade, C. N., \& Quayle, J. D. (2006). Effects of belief and logic on syllogistic reasoning: Eye-movement evidence for selective processing models. Experimental Psychology, $53,77-86$.

Bettinghaus, E., Miller, G., \& Steinfatt, T. (1970). Source evaluation, syllogistic content, and judgments of logical validity by highand low-dogmatic persons. Journal of Personality and Social Psychology, 16, 238-244.

Chaiken, S., \& Maheswaran, D. (1994). Heuristic processing can bias systematic processing: Effects of source credibility, argument ambiguity, and task importance on attitude judgment. Journal of Personality and Social Psychology, 66, 460-473.

Chater, N., \& Oaksford, M. (1999). The probability heuristics model of syllogistic reasoning. Cognitive Psychology, 38, 191-258.

Copeland, D. E., \& Radvansky, G. A. (2004). Working memory and syllogistic reasoning. The Quarterly Journal of Experimental Psychology, 57A(8), 1437-1457.

DeBono, K. G., \& Harnish, R. J. (1988). Source expertise, source attractiveness, and the processing of persuasive information: A functional approach. Journal of Personality and Social Psychology, 55, 541-546.

Demeure, V., Bonnefon, J. F., \& Raufaste, E. (2009). Politeness and conditional reasoning: Interpersonal cues to the indirect suppression of deductive inferences. Journal of Experimental Psychology. Learning, Memory, and Cognition, 35, 260-266.

Evans, J. St. B. T. (2002). Logic and human reasoning: An assessment of the deduction paradigm. Psychological Bulletin, 128, 978996.

Evans, J. St. B. T. (2006). The heuristic-analytic theory of reasoning: Extension and evaluation. Psychonomic Bulletin \& Review, 13, 378-395. 
Evans, J. St. B. T. (2007). On the resolution of conflict in dual process theories of reasoning. Thinking and Reasoning, 13, 321-339.

Evans, J. St. B. T., Barston, J. L., \& Pollard, P. (1983). On the conflict between logic and belief in syllogistic reasoning. Memory \& Cognition, 11, 295-306.

Evans, J. St. B. T., \& Curtis-Holmes, J. (2005). Rapid responding increases belief bias: Evidence for the dual-process theory of reasoning. Thinking and Reasoning, 11, 382-389.

Evans, J. St. B. T., Handley, S. J., \& Harper, C. N. J. (2001). Necessity, possibility, and belief: A study of syllogistic reasoning. The Quarterly Journal of Experimental Psychology, 54A, 935-958.

Evans, J. St. B. T., Handley, S. J., Harper, C. N. J., \& Johnson-Laird, P. N. (1999). Reasoning about necessity and possibility: A test of the mental model theory of deduction. Journal of Experimental Psychology. Learning, Memory, and Cognition, 25, 1495-1513.

Goldsmith, R. E., Lafferty, B. A., \& Newell, S. J. (2000). The impact of corporate credibility and celebrity credibility on consumer reaction to advertisements and brands. Journal of Advertising, $29,43-54$.

Gordon, R., \& Bryant, K. (2010). You don't act like you trust me: Dissociations between behavioral and explicit measures of source credibility judgment. Manuscript submitted for publication.

Gordon, R., Franklin, N., \& Beck, J. (2005). Wishful thinking and source monitoring. Memory \& Cognition, 33, 418-429.

Hadjichristidis, C., Handley, S. J., Sloman, S. A., Evans, J St B T, Over, D. E., \& Stevenson, R. J. (2007). Iffy beliefs: Conditional thinking and belief change. Memory \& Cognition, 35, 2052-2059.

Hilton, D. J., Kemmelmeier, M., \& Bonnefon, J. F. (2005). Putting ifs to work: Goal-based relevance in conditional directives. Journal of Experimental Psychology: General, 134, 388-405.

Johnson-Laird, P. N. (2006). How we reason. Oxford: Oxford University Press.

Johnson-Laird, P. N., \& Bara, B. G. (1984). Syllogistic inference. Cognition, 16, 1-61

Johnson-Laird, P. N., \& Byrne, R. M. J. (1989). Only reasoning. Journal of Memory and Language, 28, 313-330.

Kilpatrick, S. G., Manktelow, K. I., \& Over, D. E. (2007). Power of source as a factor in deontic inference. Thinking and Reasoning, 13, 295-317.

Klauer, K. C., Musch, J., \& Naumer, B. (2000). On belief bias in syllogistic reasoning. Psychological Review, 107, 852-884.

Mills, J., \& Jellison, J. M. (1967). Effect on opinion change of how desirable the communication is to the audience the communicator addressed. Journal of Personality and Social Psychology, 6, 98 101.

Newstead, S. E., Handley, S. J., \& Buck, E. (1999). Falsifying mental models: Testing the predictions of theories of syllogistic reasoning. Journal of Memory and Language, 27, 344-354.

Petty, R. E., \& Briñol, P. (2008). Persuasion: From single to multiple metacognitive processes. Perspectives on Psychological Science, $3,137-147$.

Petty, R. E., \& Wegener, D. T. (1998). Attitude change: Multiple roles for persuasion variables. In D. Gilbert, S. Fiske, \& G. Lindzey (Eds.), The handbook of social psychology (4th ed.). New York: McGraw-Hill.

Pornpitakpan, C. (2004). The persuasiveness of source credibility: A critical review of five decades' evidence. Journal of Applied Social Psychology, 34, 243-281.

Priester, J. R., \& Petty, R. E. (1995). Source attributions and persuasion: Perceived honesty as a determinant of message scrutiny. Personality and Social Psychology Bulletin, 21, 637654.

Radvansky, G. A., \& Copeland, D. E. (2004). Reasoning, integration, inference alteration, and text comprehension. Canadian Journal of Experimental Psychology, 58, 133-141.

Rhine, R., \& Severance, L. (1970). Ego-involvement, discrepancy, source credibility, and attitude change. Journal of Personality and Social Psychology, 16, 175-190.

Roberts, M. J., Newstead, S. E., \& Griggs, R. A. (2001). Quantifier interpretation and syllogistic reasoning. Thinking and Reasoning, 7, 173-204.

Sparks, J. R., \& Rapp, D. N. (in press). Readers' reliance on source credibility in the service of comprehension. Journal of Experimental Psychology: Learning, Memory, and Cognition.

Stanovich, K. E., \& West, R. F. (1997). Reasoning independently of prior belief and individual differences in actively open-minded thinking. Journal of Educational Psychology, 89, 342-357.

Stevenson, R. J., \& Over, D. E. (2001). Reasoning from uncertain premises: Effects of expertise and conversational context. Thinking and Reasoning, 7, 367-390.

Stupple, E. J. N., \& Ball, L. J. (2008). Belief-logic conflict resolution in syllogistic reasoning: Inspection-time evidence for a parallelprocess model. Thinking and Reasoning, 14, 168-181.

Tormala, Z. L., Briñol, P., \& Petty, R. E. (2006). When credibility attacks: The reverse impact of source credibility on persuasion. Journal of Experimental Social Psychology, 42, 684-691. 